\section{Der „Economist“ mag keine Senioren}

Eine der führenden Wirtschaftszeitungen der Welt verbreitet negative Stereotypen und Vorurteile gegenüber Älteren.

— In einer elektronischen Suche des digitalen Archivs der Wirtschaftszeitschrift „Economist“ analysierte man Artikel, die sich mit den Themen Pensionen, demografische Entwicklung und Politik im Zeichen einer älter werdenden Gesellschaft beschäftigten. Nur 12\% der Artikel bezeichneten diese Entwicklung als nützlich, $64 \%$ dagegen als Belastung für die Gesellschaft. $24 \%$ vermittelten einen neutralen Eindruck.

In den negativ eingestellten Artikeln fand man Formulierungen wie „demografische Zeitbombe“, ,explodierende Gesundheitskosten bei älteren Leuten“, „zusammenbrechende Pensionsfonds" und „schwächliche Mitglieder der Gesellschaft ohne Beitragsleistungen“.

\section{Kommentar}

Trotz des hohen Anspruchs und der offensichtlichen Qualität der Berichterstattung bewegt sich das Blatt beim Thema der alternden Gesellschaft überwiegend in den dumpfen Abgründen von Vorurteilen und vernachlässigt es, auch auf die Chancen des Phänomens der alternden Gesellschaft hinzuweisen, das in seinen Gesamtauswirkungen noch keineswegs klar ist. Insofern befindet sich das Hochklasseblatt in bester Gesellschaft mit bierdimpfligen Stammtischen. Wahrscheinlich hat lediglich der eigene Qualitätsanspruch bislang Formulierungen wie „nutzlose Esser“ verhindert.

H. S. FüEßL =

\section{- R. Martin et al.}

Retrospective analysis of attitudes to ageing in the Economist: apocalyptic demography for opinion formers? BMJ 339 (2009) 7735, 1435-1436

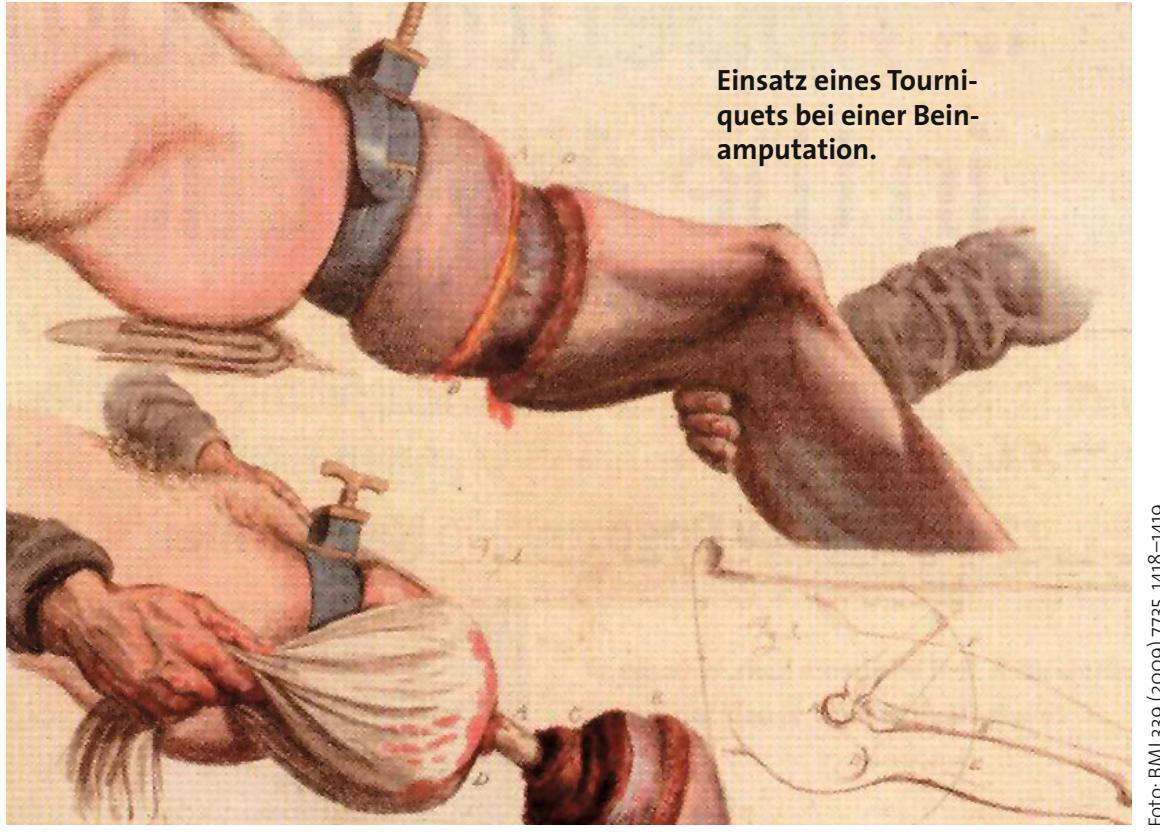

\title{
Nützliche Idee, die keiner nutzte
}

Bis zum Zeitalter der Anästhesie waren Amputationen wahre Martyrien für die betroffenen Patienten und eine Herausforderung für die Psyche des Feldschers. Dabei wären auch damals schmerzfreie Amputationen von Extremitäten möglich gewesen.

_ Über Jahrhunderte wurden die unerträglichen Leiden bei Amputationen akzeptiert, weil man wusste, dass ein Belassen einer zerschmetterten Extremität nahezu unausweichlich den Tod bedeutete. Dies erklärte wahrscheinlich, dass über die Schmerzen der so „behandelten“ Patienten in den alten Lehrbüchern der Chirurgie kaum etwas steht.

Der einzige Weg, die Schmerzen so gering wie möglich zu halten, bestand in der Schnelligkeit des Chirurgen beim Eingriff.

Gerade die Schnelligkeit, mit der die Amputation ausgeführt wurde, verhinderte möglicherweise die Entdeckung einer schmerzfreien Amputation. Tourniquets wurden nämlich bereits damals unmittelbar vor der Amputation proximal an der Amputationsstelle angebracht, um den Blutverlust zu minimieren und die extrem schmerzhafte Kauterisierung zu vermeiden. Hätte man den Tourniquet 30 Minuten lang belassen, so wäre es durch die gestörte Nervenfunktion im Rahmen der Ischämie zu einer vollständigen Anästhesie der Extremität gekommen. Jeder von uns hat das schon einmal erfahren, wenn „der Fuß einschläft".

Man fragt sich, warum angesichts Hunderttausender Amputationen im Lauf der Historie dieses Phänomen nicht wenigstens einmal durch Zufall entdeckt wurde. Es finden sich tatsächlich in den Jahren 1637 und 1679 zwei Beschreibungen von Chirurgen, die eine weitgehende Aufhebung der Schmerzempfindung durch die starke Kompression des Tourniquets beschreiben.

\section{Kommentar}

Wahrscheinlich hat man sich aber darüber zu wenig Gedanken gemacht, da Chirurgen zu dieser Zeit schnell arbeitende Operationshandwerker waren, die in aller Regel mit großer Geschwindigkeit und unter dem Stress einer laufenden Schlacht arbeiten mussten. Es war einfach keine Zeit, um über Zufallsentdeckungen und eigenartige Phänomene nachzudenken. Was lehrt uns diese Geschichte heute noch? Für den Fortschritt in der Medizin ist es notwendig, dass nicht alle in der klinischen Praxis und der Routine ertrinken, sondern es auch Ärzte gibt, die sich über Phänomene wundern, Fragen stellen, sich zurücklehnen und viel nachdenken.

H. S. FÜEßL =

- S. Shuster et al.

(sam@shuster.eclipse.co.uk), Painless amputation: history of a discovery that wasn't made. BMJ 339 (2009) 7735, 1418-1419 